\title{
A Reservation Scheme Satisfying Bandwidth QoS Constraints for Ad-hoc Networks
}

\author{
Llorenç Cerdà $^{1}$, Michael Voorhaen ${ }^{2}$, Rafael Guimarães ${ }^{1}$, \\ José-M Barceló $^{1}$, Jorge García ${ }^{1}$, and Chris Blondia ${ }^{2}$ * \\ 1 Technical University of Catalonia \\ Computer Architecture Dept. \\ Jordi Girona 1-3, E-08034 Barcelona, Spain \\ \{llorenc, rafael.guimaraes, joseb, jorge\}eac.upc.es \\ ${ }^{2}$ University of Antwerp \\ Dept. Mathematics and Computer Science \\ Middelheimlaan 1, B-2020 Antwerpen, Belgium \\ \{michael.voorhaen, chris.blondia\}@ua.ac.be
}

\begin{abstract}
Achieving QoS (Quality of Service) in Mobile Ad-hoc NETworks (MANET) has been a research topic in the last years. In this paper we describe a QoS reservation mechanism for Routing Ad-hoc Networks. The mechanism is targeted for sources requiring a bandwidth allocation. The mechanism is based on the knowledge of the bandwidth requirements of the neighbors of a node and the interferent nodes in the cover area of each node. We describe as the protocol could be integrated in AODV and OLSR. We also give simulation results showing the advantages of our reservation scheme.
\end{abstract}

\section{Introduction}

MANETs (Mobile Ad-hoc NETworks) have characteristics such as flexibility, fast and easy deployment, robustness which make them an interesting technology for military, public safety, emergency and disaster applications. Providing QoS (Quality of Service) in a MANET is, however, a difficult task because: (i) the capacity of the physical links is variable depending on factors such as the distance, signal to noise ratio, interference, etc, (ii) the transmission media is shared between different nodes that have to be synchronized, (iii) MANET nodes are generally mobile and the network topology may change, and (iv) high signaling overhead due to the recovery of already hard-QoS reservations may be a problem due to the scarce transmission resources.

\footnotetext{
* This work was supported by the Ministry of Edu. of Spain under grant CICYT TIC-20010956-C04-01, by the Generalitat de Catalunya under grant CIRIT 2001-SGR-00226, by the Fund for Scientific Research Flanders under Scientific Research Community Broadband communication and multimedia services for mobile users, by DWTC Belgium under project IAP P5/11 MOTION (Mobile Multimedia Communication Systems and Networks), by IWT under project 020152-End-to-end QoS in IP based Mobile Networks, by the European NoE EuroNGI and by the European project WIDENS.
} 
The provisioning of QoS in a MANET involves the inter-working of several mechanisms spanning from the physical layer to the application layer. We center our work on the mechanisms related with the network layer: resource reservation, signaling and routing.

There are several QoS frameworks for MANETs proposed in the literature addressing some of the aspects for QoS support. Authors of [12] present a framework called FQMM (Flexible Quality of Service Model) that combines a reservation procedure for high priority traffic with a service differentiation for low-priority traffic. However, this hybrid provisioning scheme does not take into account the characteristics of MANETs and all the drawbacks of the IntServ and DiffServ remain. Other proposals are less general and address some of the aspects to be taken into account in a QoS framework for MANETs. INSIGNIA, see [3], is an in-band signaling protocol designed explicitly for MANETs which must be integrated with an ad-hoc routing protocol. CEDAR, see [9], is a protocol proposed to reduce the control overhead by defining a backbone and MMWN, see [8], is defined for cluster networks. A reservation scheme with AODV can be found in [13]. Another QoS approach based on measurements is presented in [2].

In this paper we treat the problem of achieving a reservation taking into account the available bandwidth in a coverage area and the traffic generated and forwarded by the neighbors and interferent MNs in that coverage area. Furthermore, we apply our reservation policy to AODV ([6]) and OLSR ([10]) routing protocols, although the reservation scheme can be applied to other ad-hoc routing protocols. The results show the feasibility of our scheme for guaranteeing the QoS requirements. Our reservation scheme works well for low mobility scenarios. Further analysis to optimize the reservation scheme under high mobility scenarios still is a current research topic.

\section{Bandwidth QoS Constraint}

Through this paper we shall assume the following:

- Without loss of generality, there are two traffic classes: with QoS and best effort.

- The MAC is able to isolate traffic classes such that QoS connections have priority over best effort, e.g. by using $802.11 \mathrm{e}$.

- We use a bandwidth reservation scheme (e.g. peak rate allocation) subject to the following QoS constraint:

The occupancy of the wireless media by the QoS connections observed at any $M N$ (i.e. transmitted or received by the MN antenna) is $\leq Q$ bps. I.e. the occupancy of the QoS traffic competing for the same shared media at any $M N$

$$
\text { is } \leq Q \text { bps. }
$$

The parameter $Q$ could be dimensioned such that delays are acceptable for QoS connections.

- A Call Admission Control (CAC) is used to block new QoS connections if the QoS constraint cannot be fulfilled.

We shall refer to the QoS traffic generated or in transit at $M N_{i}$ as the bandwidth reservation $\left(x_{i}\right)$ at a $M N_{i}$. I.e. if $r_{i j}$ is the amount of QoS traffic sent from $M N_{i}$ to $M N_{j}$, then: 


$$
x_{i}=\sum_{j \in \mathcal{N}_{i}} r_{i j}
$$

Where $\mathcal{N}_{i}$ is the set of neighbor MNs of $M N_{i}$, i.e. $\left\{M N_{j} \mid j \in \mathcal{N}_{i}\right\}$ is the set of nodes in range with $M N_{i}$.

We define the maximum available bandwidth $M A B_{i}$ for QoS traffic at $M N_{i}$ as:

$$
M A B_{i}=Q-x_{i}-\sum_{j \in \mathcal{N}_{i}} x_{j}
$$

Therefore, the QoS constraint previously defined can be formulated as:

$$
\text { QoS constraint: } M A B_{i} \geq 0, \forall i \text {. }
$$

Note that the previous formulation of the QoS constraint would not be accurate for a MAC as 802.11 using RTS/CTS. This is because all nodes receiving not only RTS but also CTS are silent. Therefore, wireless media occupancy at a MN should be defined not only by the traffic transmitted by the neighbors, but also by the traffic received by them. Assume for instance the example shown in Fig. 1. In this figure there is only one ongoing QoS connection of $Q$ bps from $M N_{1}$ to $M N 2$. Thus, using equations (1) and (2) we have: $x_{1}=Q, x_{2}=x_{3}=x_{4}=0$, and: $M A B_{1}=M A B_{2}=0, M A B_{3}=M A B_{4}=Q$. In case $M N_{4}$ accepts a reservation of $Q$ bps for $M N_{3}$, then $M A B_{1}=M A B_{2}=M A B_{3}=$ $M A B_{4}=0$, thus the QoS constraint is satisfied. However, $M N_{3}$ cannot receive packets from $M N_{4}$, since $M N_{3}$ is blocked by the CTS signals received from $M N_{2}$ (this is the well-known exposed node problem that occurs when using RTS/CTS).

Note also that even if $M A B_{3}=Q, M N_{3}$ cannot accept new reservations due to the traffic constraint that $M N_{2}$ imposes on $M N_{3}$ (due to the well known hidden node problem that occurs in wireless networks). However, since our QoS constraint takes into account the traffic transmitted by the neighbors, it correctly captures this limitation. Assume for instance $M N_{3}$ accepting a reservation of $Q$ bps for $M N_{4}$. Then, the MNs would have: $x_{1}=Q, x_{2}=0, x_{3}=Q, x_{4}=0$, thus: $M A B_{1}=0, M A B_{2}=-Q$, $M A B_{3}=M A B_{4}=0$, so, the QoS constraint would not be satisfied.

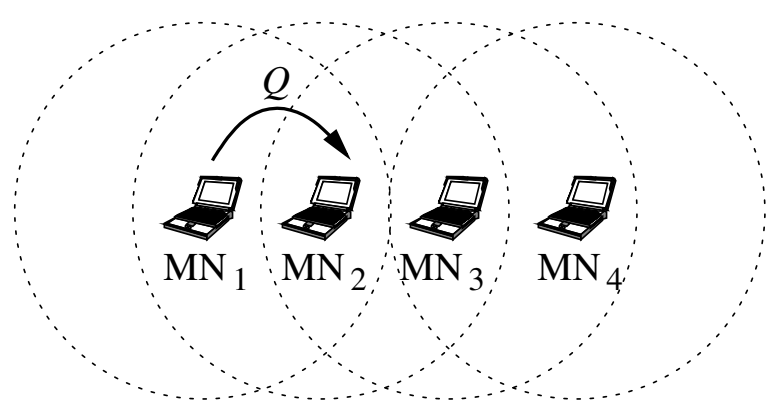

Fig. 1. Hidden node problem in QoS reservations. 


\section{Reservation Approach}

We shall assume that connections requiring QoS use a reservation mechanism at the connection setup. This consists in the source sending a Reservation Request to the destination, which in turn sends back a Reservation Reply to the source if the reservation could be allocated.

Furthermore, we shall also assume that a Call Admission Control (CAC) is used to block new QoS connections if the QoS constraint cannot be fulfilled: i.e. a MN only accepts a Reservation Request if the QoS constraint can be satisfied.

We define the available bandwidth $A B_{i}$ to allocate new reservations at $\mathrm{MN}_{i}$ as:

$$
A B_{i}=\min \left\{M A B_{i}, M A B_{j}\right\}, j \in \mathcal{N}_{i}
$$

We shall use the notation $M N_{i} \rightarrow M N_{j}$ to denote two consecutive MNs belonging to the path to be reserved for a new QoS connection. Suppose that a new QoS connection of $r$ bps has to be established. We claim that if the path to be reserved does not follow unnecessary jumps (i.e. if $\cdots M N_{i} \rightarrow M N_{j} \rightarrow M N_{k} \cdots$, then $M N_{i}, M N_{k} \in \mathcal{N}_{j}, M N_{l} \notin$ $\left.\mathcal{N}_{j}, \forall l \neq i, j, k\right)$, then, the QoS constraint given by (3) is satisfied if the following CAC conditions hold:

- If the QoS connection is generated at $M N_{i}$ :

- Accept if the destination is a neighbor and $A B_{i} \geq r$.

- Accept if the destination is not a neighbor and $A B_{i} \geq 2 r$.

- If the QoS connection is generated at another MN (transit traffic):

- Accept if the destination is a neighbor and $A B_{i} \geq 2 r$.

- Accept if the destination is not a neighbor and $A B_{i} \geq 3 r$.

- Otherwise the reservation request cannot be accommodated.

Note that this conditions should be fulfilled in every node along the path.

Proof. Assume that the Reservation Request is accepted and use (2) and (4) to verify that the QoS constraint is satisfied.

For instance, suppose the case when the new connection of $r$ bps is generated at $M N_{1}$ and the destination is not one of its neighbors (see Fig. 2). Assume that the CAC accepts this connection, and the next hop is the node $M N_{2}$ shown in the figure. Then, $x_{1}$ and $x_{2}$ will be increased by $r$. Thus, the $M A B$ of $M N_{1}, M N_{2}$ and all their common neighbors will be decreased by $2 r$. Since $A B_{i} \geq 2 r$, from (4), $M A B_{i} \geq 0, \forall i$.

The formerly described CAC may be further improved. Consider for instance that the node imposing the minimum $M A B$ in the previous example is a neighbor of $M N_{1}$ but not of $M N_{2}$. In this case, the $A B$ may be reduced by less than $2 r$, thus the condition $A B_{i} \geq 2 r$ may be too restrictive. However, in order to introduce this optimization, the nodes would not only need to know their neighbors, but also the coverage relation among them. 


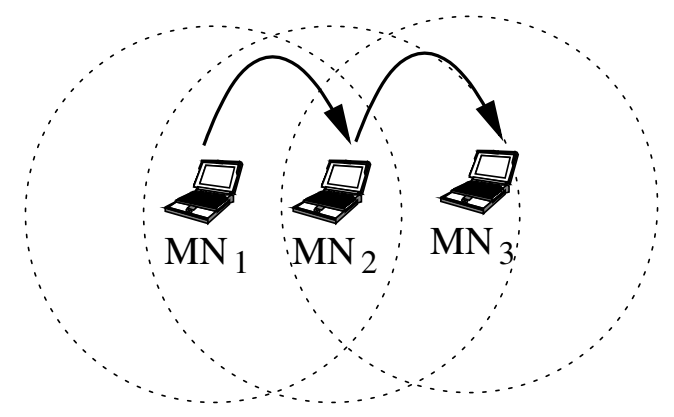

Fig. 2. Example of a connection generated at $M N_{1}$ with destination not a neighbor of $M N_{1}$.

\section{Implementation}

The reservation scheme described in the previous sections requires that each $M N_{i}$ knows two quantities from their neighbors $\mathcal{N}_{i}$ : their reservation $\left(x_{j}, j \in \mathcal{N}_{i}\right)$ and their maximum available bandwidth $\left(M A B_{i}, j \in \mathcal{N}_{i}\right)$. This could be implemented by means of each $M N_{i}$ broadcasting $H E L L O$ packets with $x_{i}$ and $M A B_{i}$.

In the following we describe how to integrate our reservation scheme in the AODV [6] and OLSR ad-hoc [10] routing protocols.

\subsection{Integration in AODV}

For the AODV protocol we propose the following modifications to implement the reservation scheme:

(i) AODV HELLO messages are modified such that each $M N_{i}$ advertises $x_{i}$ and $M A B_{i}$ to their neighbors.

(ii) Each $M N_{i}$ collects the QoS messages from their neighbors to compute $A B_{i}$ according to (4).

(iii) QoS Reservation Request and Reply messages are integrated in AODV as described in [5]: the bandwidth reservation is included in a Route Request (RREQ) message as an extension object. The RREQ QoS extensions include a session-ID to identify the flows together with the Source and Destination addresses.

(iv) Upon receiving a RREQ, intermediate MNs apply the CAC algorithm described in Sect. 3. If the reservation is accepted, the RREQ is forwarded, and it is discarded otherwise. However, reservation is only done when the RREP is received (see Fig. 3). Opposite to AODV, if an intermediate MN has a route to a destination, this MN should not answer with a route reply to the sender, since the intermediate MN does not know whether further MNs can accomplish the bandwidth reservation. In order to avoid this situation the D flag of a RREQ is activated (see [6]) indicating that only the destination can send a RREP. 


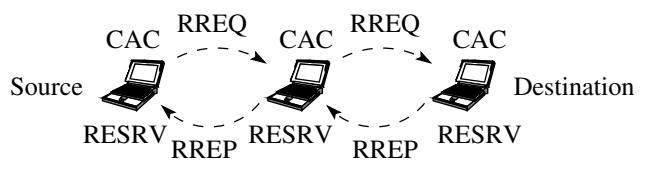

Fig. 3. Reservation procedure.

\subsection{Integration in OLSR}

The modifications we propose for OLSR to implement the reservation scheme is somehow similar to what happens for AODV, as one may notice in the following steps:

(i) OLSR HELLO messages are modified just like in AODV (see steps i and ii).

(ii) We modified the OLSR TC messages such that they also advertise $A B_{i}$ next to advertising the MPR selectors. This way each node has knowledge of the network topology and the bandwidth available in the network.

(iii) In order to find a route that meets the QoS requirements, we modified the OLSR route selection algorithm to find a shortest hop path that has enough bandwidth to meet these requirements. Since the TC messages also advertise $A B_{i}$ the originating node has enough information to decide if enough resources are available.

(iv) The reservation of the bandwidth at the intermediate nodes is done by adding the requested rate to the IP header (e.g. by using an IP option). This way an intermediate node which has not yet seen the flow will be able to allocate the bandwidth. The requested rate can be advertised for a certain amount of time, number of packets or until an ACK is received to say that the flow has been set up. This is done to make this approach robust to packet loss.

For the remainder of the paper we will call our OLSR implementation extended with the QoS signaling QOLSR. We wish to stress that our protocol has little in common with [1], only the idea of extending OLSR with QoS support. The signaling introduced in QOLSR has much in common with the INSIGNIA protocol [3], although the full feature set of INSIGNIA is not implemented since this was not necessary for the goal of this paper.

\subsection{Flow Based Routing}

Some words about flows: note these reservation schemes need a flow-id to identify ongoing QoS connections. IP look-ups are done using the pairs IP-address and Flowid. These pairs must be unique. Using IPv6 it can be used the flow label specification described in [7]. Connections are identified by the 3-tuple Flow Label, Source and Destination Address fields. Packet classifiers use the former triplet to identify which flow a particular packet belongs to. Note that packets from different flows forwarded to the same destination may follow different paths. 


\section{Simulation Results}

We have added our reservation scheme in the AODV protocol provided by the network simulator [11] and the OLSR implementation available in [4]. We shall refer as QAODV and QOLSR to our implementations. We have simulated the following scenario:

- MAC: 802.11 without RTS/CTS and link rate of 2 Mbps.

- CBR connections sending packets of size 500 bytes and rate $32 \mathrm{kbps}$.

- The QoS constraint for CBR connections is $Q=250 \mathrm{kbps}$.

- $40 \mathrm{MNs}$ randomly placed over a square $1000 \times 1000$ meters.

- MN coverage of 300 meters.

- Each pair of nodes initiates a unidirectional QoS connection staggered 15s. Thus, 20 QoS connections are initiated $(20 \times 32=640 \mathrm{kbps})$.

- The simulation time is $600 \mathrm{~s}$.

- The nodes don't move.

In the following we explain the results obtained using AODV/QAODV and OLSR/QOLSR.

\subsection{Simulation results with AODV}

Fig. 4 shows the evolution of the connections established with each protocol. Note that all connections are established with AODV but only 8 with QAODV (the others are blocked). Fig. 6 shows the distribution of the MNs and the connections that were successfully established. The figure also shows the coverage (300 meters) for one of the nodes.

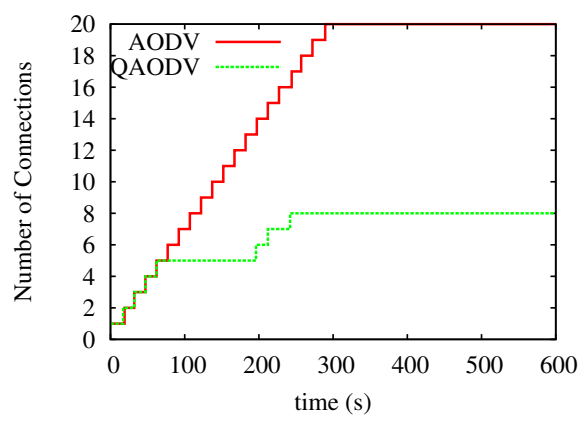

Fig. 4. Connection setup.

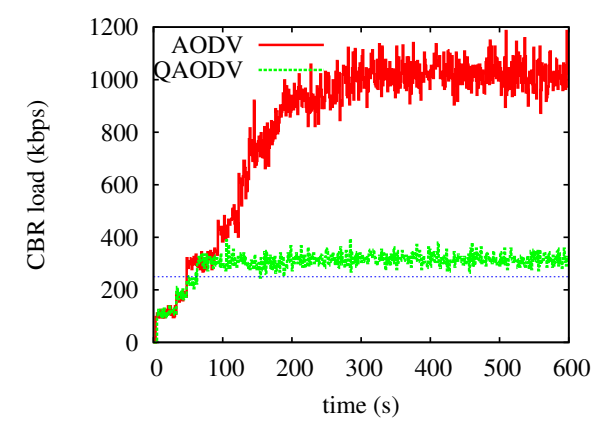

Fig. 5. Maximum occupancy.

Fig. 5 plots at each time $t$ the maximum CBR occupancy (as defined in section 2) observed by the most congested node at this moment. This occupancy is measured at each node counting the size of the frames carrying CBR packets that are seen by the node (including the collisions). This figure validates that the QoS constraint is satisfied, i.e. the maximum CBR occupancy is $\approx 250 \mathrm{kbps}$. This value is exceeded a bit among other reasons, because of collisions and headers overhead (the 500 bytes packet size 


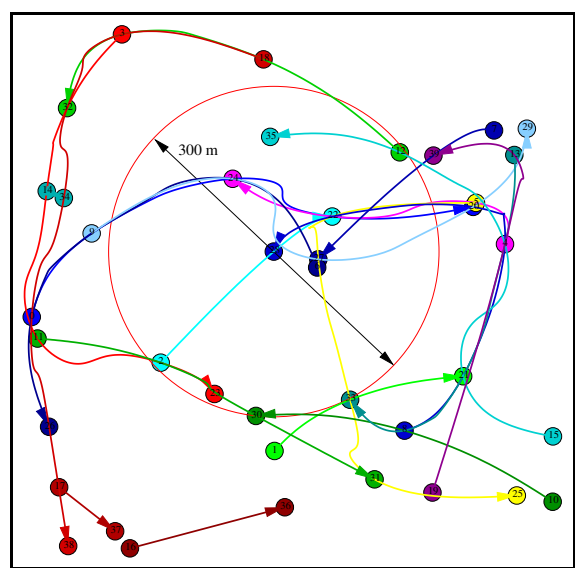

(A)

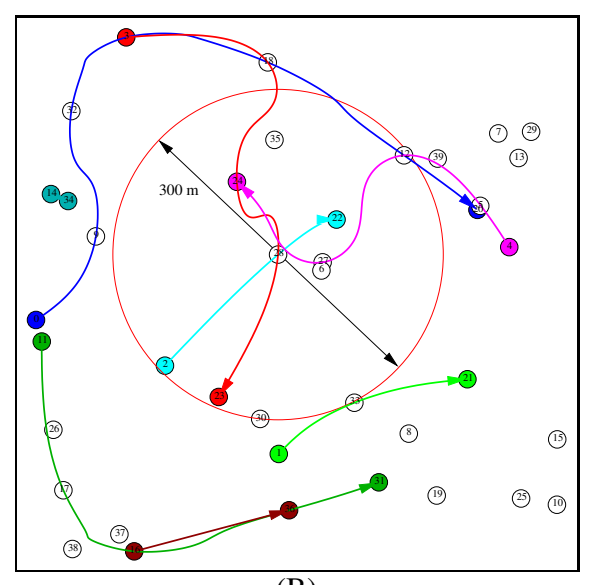

(B)

Fig. 6. Connections established using AODV (A) and QAODV (B).

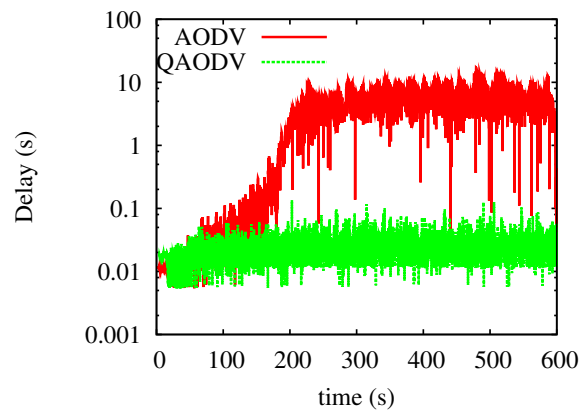

Fig. 7. Maximum delay.

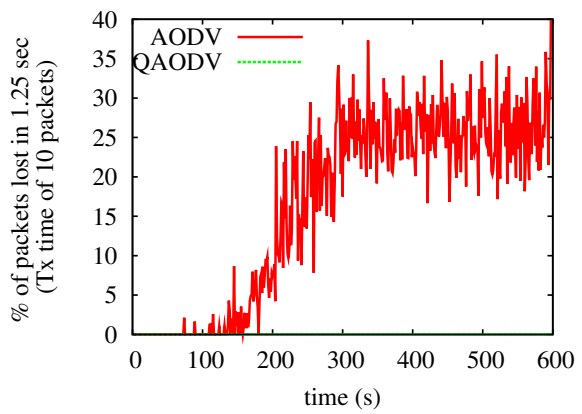

Fig. 8. Maximum loss.

does not include the IP header, neither the 802.11 header, thus, the occupancy at the MAC is in fact $250 \times 572 / 500=286 \mathrm{kbps}$ ). Thus, we conclude from Fig. 5 that the QoS constraint is satisfied.

Fig. 5 also shows that, using AODV, the MAC becomes congested at around 200 seconds (when only 14 of the 20 connections have started). This may be noticed by the fact that the CBR occupancy does not increase any more, although new connections are established. This is confirmed by Figs. 7 and 8 . The first one depicts the maximum endto-end delay of CBR packets, and the second one depicts the maximum percentage of packets lost by the connections, measured in intervals of $1.25 \mathrm{~s}$ (the transmission time of 10 packets).

It is also interesting to know how may connections are suffering from congestion. Figures 9-10 and 11 show respectively delay and loss-percentage Complementary Distribution Probability Functions (CDPF) for all the ongoing connections (loss CDPFs are not given for QAODV because no losses were detected with it). 

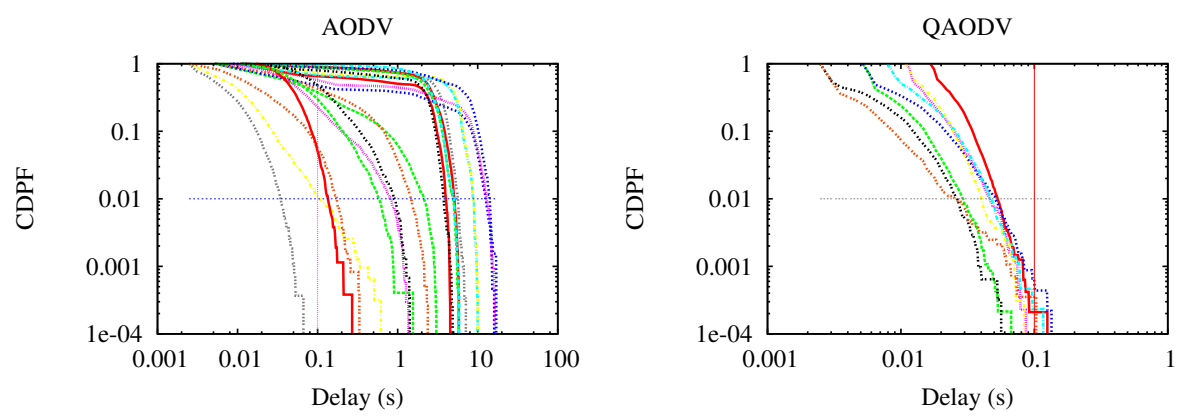

Fig. 9. CDPFs of the delay for all AODV con- Fig. 10. CDPFs of the delay for all QAODV connections. nections.

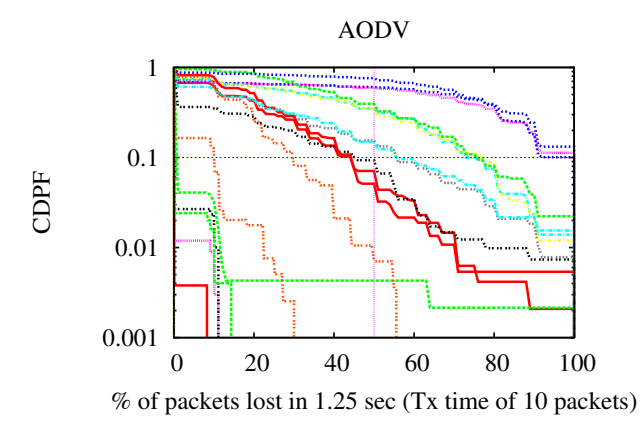

Fig. 11. CDPFs of packet loss for all AODV connections.

Fig. 9 shows that delays are huge for AODV: 11 of the 20 connections have delays higher than $2 \mathrm{~s}$ with probability higher than 30\%. On the other hand, Fig. 10 shows that all connections with QAODV have delays higher than $60 \mathrm{~ms}$ with probability lower than $0.1 \%$. Fig. 11 also shows that most of AODV connections suffer high loss rates (11 of the 20 connections have losses higher than $50 \%$ with probability higher than $5 \%$ ). Thus, we conclude that most of the ongoing AODV connections are suffering from congestion.

\subsection{Simulation results with OLSR}

The following paragraph describes the results obtained from the simulations using OLSR and QOLSR. Since OLSR is a proactive protocol we had to make sure that each node received the necessary topology information before the applications were started. We changed the parameters so that the simulation now took 700s. During the first 100 seconds no application was started, only OLSR routing messages were exchanged. This 100 second period gives OLSR ample time to exchange routing information between all nodes. We now compare the results of the last 600 s of our simulation to those from AODV. 


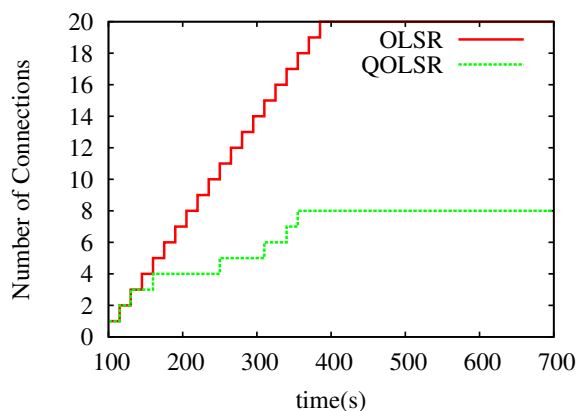

Fig. 12. Connection setup.

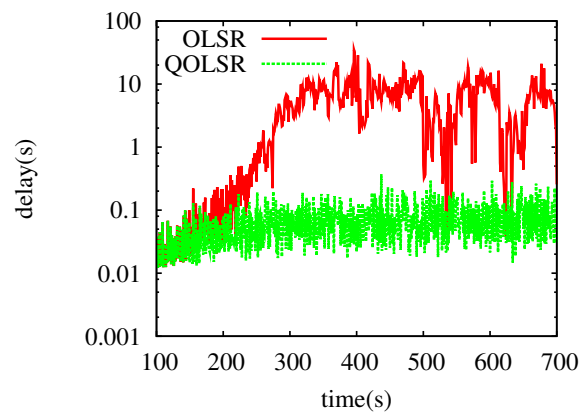

Fig. 14. Maximum delay.

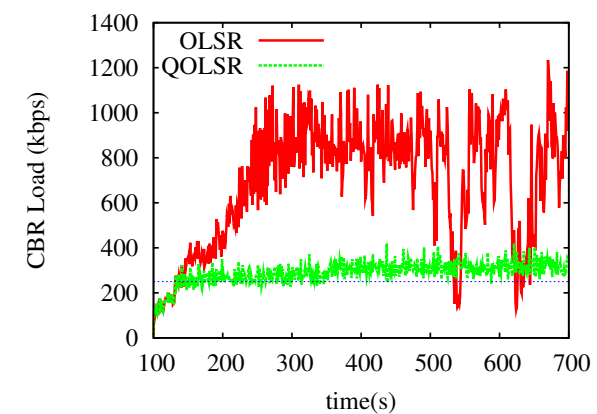

Fig. 13. Maximum occupancy.

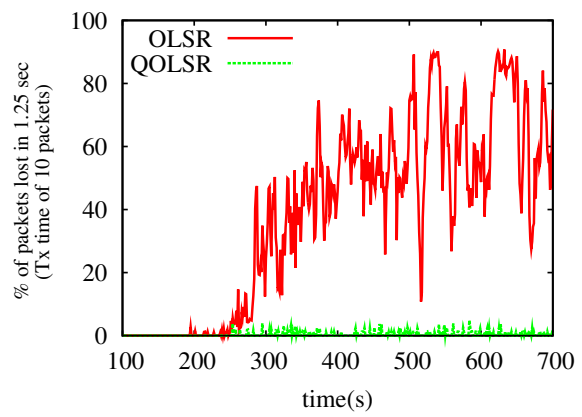

Fig. 15. Maximum loss.

Fig. 12 shows us that 8 connections are allowed to start, while the others are blocked by the CAC. However when observing the graph we notice that different applications are allowed to start by QAODV and QOLSR protocols. After carefully looking at the routes that were set up by both protocols we found that the route for some of the flows was chosen differently by them. In fact both protocols could choose between the same routes and both made an arbitrary choice (which is influenced by randomness e.g. the order of the links in the topology set of OLSR and the jitter introduced before rebroadcasting a RREQ in AODV). This different choice in routes caused that different applications were allowed to start.

Of course the previous paragraph does not prove that the CAC performed by both protocols behaves equally well since the result depends very much on the node placement and several random factors inherent to the scenario. To investigate any possible performance differences between the two protocols we ran the same simulation 400 times with both QAODV and QOLSR but each time using different node placements. Figures 20 and 21 show the results of these simulations.

As you can see QOADV allows on average 7 connections with a standard deviation of about 2 connections. QOLSR presents similar results, with an average of 7 to 8 connections and a standard deviation of 2 connections. It is interesting to notice that, although OLSR disseminates network topology using the multi-point relays (what causes only partial topology information to be spread), results are as good as in the case 


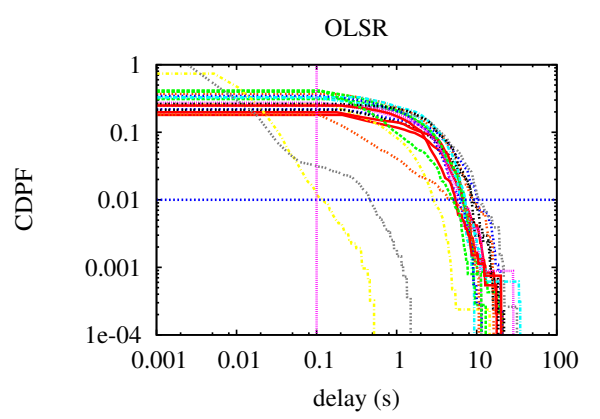

Fig. 16. OLSR delay CDPF.

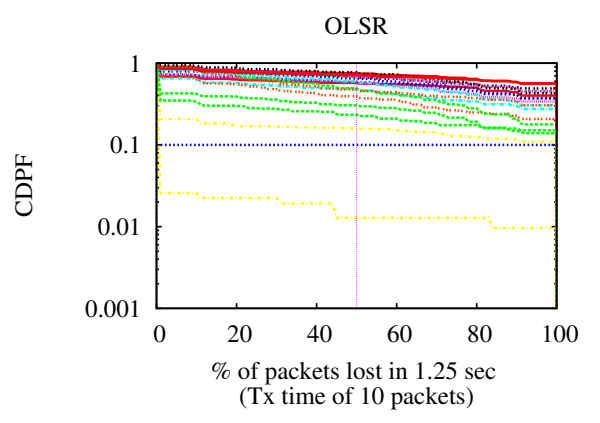

Fig. 18. OLSR loss CDPF.

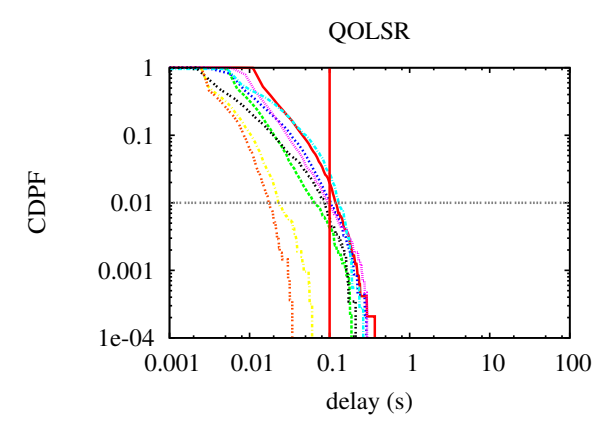

Fig. 17. QOLSR delay CDPF.

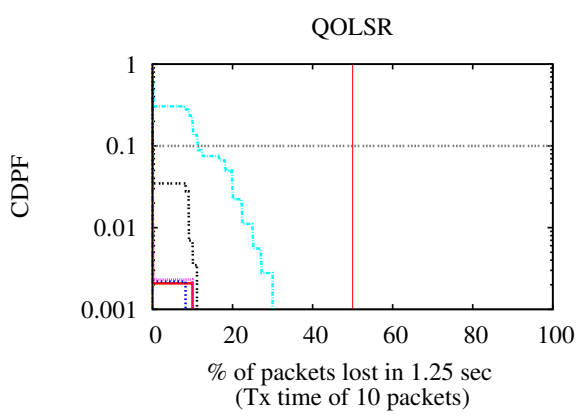

Fig. 19. QOLSR loss CDPF.

of AODV, where this restriction is not present. In fact, repeating the experiment with QAODV using RTS/CTS, we obtained an average of 11 connections. The drawback of QAODV is that some nodes may not receive the broadcast because of collisions. This is more likely to happen without RTS/CTS, due to the hidden node problem. Thus, suboptimal paths may be chosen with QAODV, reducing the number of connections.

If we compare Fig. 5 to Fig. 13 we observe the following: QOLSR performs equally well as QAODV and both succeed in guaranteeing the QoS constraint. Secondly we also notice the drops in the occupancy measured with OLSR which did not occur with AODV. This occurs when the network gets congested and some of the broadcasts that are needed to advertise the topology information to the network get lost causing some nodes not to have full topology information. Since in this case these nodes might not have a route to the destination they will drop the packets before passing them to the MAC layer. After a while the MAC will get less congested and the topology information is advertised correctly.

We can see the same behavior in Fig. 14 where the delay drops at the same time the MAC gets less congested. Compared to QOADV (Fig. 7) we see that in QOLSR (Fig. 14) the packets incur a slightly higher delay which is confirmed by Fig. 17. Fig. 16 shows us that except for two flows all the others have a $10 \%$ chance of having a $10 \mathrm{~s}$ delay. With QOLSR on the other hand the majority of the flows have end-to-end delays 

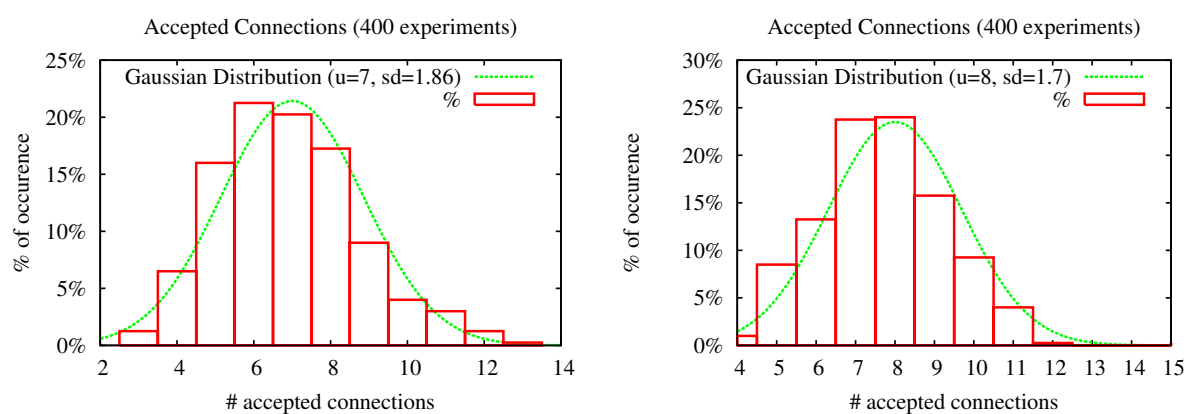

Fig. 20. \% of accepted connections by QAODV Fig. 21. \% of accepted connections by QOLSR

higher than $100 \mathrm{~ms}$ with a probability lower than $1 \%$ (Fig. 17). The remaining flows show a better behavior than the two flows that behaved best with OLSR.

Fig. 15 shows us that QOLSR is not only successful in avoiding network congestion, but also in avoiding the packet losses that occur when the network becomes congested. Compared to QOLSR, OLSR behaves much worse since it loses up to $90 \%$ of the packets at some instances. Fig. 19 shows us that QOLSR has negligible losses just like QAODV.

\section{Conclusions and further work}

In this paper we have described a bandwidth reservation scheme for ad-hoc networks that satisfies the following QoS constraint: "The occupancy of the wireless media by the QoS connections observed at any mobile node (MN) is $\leq Q$ bps". Our reservation scheme only requires that MNs know the reservation and maximum available bandwidth of their neighbors. These quantities can be easily advertised by means of hello packets. We also give the CAC rules that MNs should apply to new connections requiring QoS.

We have described how to integrate our reservation scheme in the AODV and OLSR ad-hoc routing protocols and we have implemented them with the ns simulator. We have have simulated AODV and OLSR with and without our reservation scheme. The results obtained for both protocols are similar. The following items summarize our findings:

- Ad-hoc networks can easily become congested by QoS traffic (opposite to TCP, this kind of traffic typically doesn't have congestion control mechanisms).

- Congestion can easily extend to most of the network introducing high delays and losses, thus, damaging most of the connections having QoS requirements.

- Our reservation scheme provides a feasible way to avoid congestion, thus, guaranteeing QoS requirements to ongoing connections.

- Confronting our reservation scheme integrated in AODV and OLSR, we have observed that AODV allows on average almost the same number of connections than OLSR.

Further work: In the simulations carried out in this paper we have used static MNs (without movement). If MNs move, they may enter in coverage with new MNs, producing QoS violation (i.e. breaching the QoS constraint). A mechanism in needed to cope 
with this situation. For instance, the MNs receiving hello packets from a new $\mathrm{MN}$ such that a QoS violation occurs, may send a Route Error to some connections such that they look for another path that fulfills the QoS constraint. Other reasons may produce QoS violations, e.g. due to transient periods, or due to the establishment of a path having unnecessary jumps inside the coverage of another MN.

Another problem arises when a link is broken and a set of connections that traverse that node loose the reserve path to their destination. A reservation recovery must be initiated on the nodes whose flows have lost the QoS reservations. Furthermore, a mechanism to free the already reservations in the broken path is needed. This mechanism may use timers that free a reservation in a node if the interval of time after forwarding a packet belonging to a flow is higher than certain value.

\section{References}

1. I. Gawedzki, A. Munaretto, K. Al Agha, and H. Badis. QOLSR: QoS with the OLSR protocol. http://qolsr.lri.fr/.

2. M. Kazantzidis, M. Gerla, and S. Lee. RFC 3697: Permissible throughput network for adaptative multimedia in AODV MANETs. In IEEE ICC 2001, 2001.

3. S. Lee, G. Ahn, and A. T. Campbell. Improving UDP and TCP performance in mobile ad hoc networks with INSIGNIA. In IEEE Communications Magazine, June 2001.

4. Naval Research Laboratory (NRL). NRLOLSR Implementation. http://pf.itd.nrl.navy.mil/projects.php?name=olsr.

5. C. Perkins and E. Belding-Royer. Quality of Service for Ad hoc On-Demand Distance Vector Routing (work in progress), Oct 2003. draft-perkins-manet-aodvqos-02.txt.

6. C. Perkins and S. Daas E. Belding-Royer. RFC 3561: Ad hoc On-Demand Distance Vector Routing, Jul 2003.

7. J. Rajahalme, A. Conta, B. Carpenter, and S. Deering. RFC 3697: IPv6 Flow Label Specification, March 2004.

8. R. Ramanathan and M. Streenstrup. Hierarchically-organized, multihop wireless networks for quality of service support. In ACM Mobile Networks and Applications, Vol 3, $N^{o} 1$, June 1998.

9. R. Sivakumar, P. Sinha, and V. Bharghavan. CEDAR: A COre-Extraction Distributed Ad Hoc Routing Algorithm. In IEEE JSAC, Special Issue on Ad Hoc Networks, Vol 17, $N^{o} 8$, August 1999.

10. P. Jacquet T. Clausen. RFC 3626: Optimized Link State Routing Protocol (OLSR), Oct 2003.

11. UCB/LBNL/VINT. The Network Simulator ns-2. http://www.isi.edu/nsnam/ns.

12. H. Xiao, W. G. Seah, A. Lo, and K. C. Chua. A flexible quality of service model for mobile ad hoc netowrks. In Proceedings of the IEEE Vehicular Technology Conference, Tokio, Japan, May 2000.

13. Q. Xue and A. Ganz. Ad hoc QoS on-demand routing (AQOR) in mobile ad hoc networks. Journal of Parallel and Distributed Computing, (63), 154-165, 2003. 